\title{
Sachregister zu Band 88
}

Die fett gedruckten Zahl Bb. = Buch

Ablatio retinae $46 \mathrm{ft} ., 342$

falciformis congenita 347

doppelseitige - mit symmetri-schem Oralriß bei 2 emmetropen Brüdern 154

- $\quad$ und Unfall 153Adaptation 262

Adiesche Krankheit und Pu-

pillotonie 20 Albinismus 118 Amyloide Degeneration der

Bindehaut 281 Anatomie 132 (Bericht) Aneurysma arterio-venosum

der Netzhaut 52 Angioide Pigmentstreifen 45,

63 Angiomatosis retinae 52

- intramedullaris 179Angiopathia retinae, trauma-

tische 33

- $\quad$ zerebrale Symptome bei juve-niler - -62

Angiospasmus, Augenhinter-

grund bei - 41 Aniridie 117 Anophthalmus 115 Anthropologie 127 Aphakieb. Netzhautabhebung

342 Arachnodaktylie und Ektopia

lentis 322 Area Martegiani 1 Arteria centralis retinae, Ver-

schluß der - 41 Arteria hyaloidea, persistie-

rende 119 Arteriosklerose, Retinopathie

bei - 39 Astigmatismus, Beziehungen des

Hornhaus-A. zum Gesamt-A.

331 Augenbinde, Lastex-A. 29 Augenhintergrund, Gefäßver-

änderungen am -285

- $\quad$ Mikrometrie des -329

- Veränderungen des - bei tube-

röser Hirnsklerose 15 Augenlider s. Lider Ausgewählte Schriften und

Reden 295 (Bb.) Autokeratoplastik nach Morax

177

Bindehaut s. Conjunctiva Blaulichtbestrahlung 151 Bluterkrankungen, Veränderungen der

Netzhaut bei - 42 Brille, Geschichte der - 131

en bedeuten Eigenarbeiten. besprechungen.

Brillenkunde 328ft. Bulbus, Lageveränderungen des - im fetalen Leben 112

Canalis hyaloideus mit Area Martegiani und symmetrisches Foramen hyaloideae posterius 1

Carotinverwendung bei Horn-hautverpflanzung 107

Chalazion, Pseudotumor der Lider unter dem Bilde des - 64

Chalcosis retinae 34

Chinin, Schädigung der Retina durch -34

Cholesterinkristalle in der Lin-se 281

Chorioidea, Pathologieder-30ff. 
- $\quad$ Beobachtungen an der vorderenGlaskörpergrenzhaut bei Ab-hebung der - -168

- Sarkom der - 185 Coactatio conjunctiva mit Ger-

manin behandelt 174 Commotio retinae 34 Conjunctiva, Struktur des Epi-

thels der $\square-\square 171$

endogene Affektionen der - 291

amyloide Degeneration der - 281

Cornea, Entwicklung der - 112

Anomalien der - 136

angeborene Anomalien der - 117

- $\quad$ Lupus erythematodes der - 292 - Carotinverwendung bei Ver-

pflanzung der - 107 Cysticercus retinae 35

Dacryocystitis, angeborene 122 Dampfkauterisation einer Epi-

thelwucherung in der vorderen

Kammer 315 Dermoide des Augapfels 124 Diabetes, Retinopathie bei - 39 Dioptrik 328ft.

Diplobazillus Morax 178 Druck, Beziehungen des intra-

kraniellen - zu der Zirkulation

der Netzhaut 287

Eidetische Fähigkeiten 273 í Einschlußblennorrhoe 164 Eklampsie, Retinopathie bei ---40

Ektopia lentis 120

- $\quad$ und Arachnodaktylie 322 .Elektrokauter zur Benandlung

von Lidgeschwülsten 166 Endophlebitis, Netzhautverän-derungen bei - 36

350

Sachregister zu Band 88.

Endotheliom der Sehnerven-

scheiden 178 Entropium 122 -- Entfernung des Tarsalteiles des

Orbikularis zur Bekämpfung des

- 100 Entwicklungsgeschichte des

Auges 112 Epithelwucherung in der vor-

deren Kammer, durch Dampf-

kauterisation geheilt 315 Erbkranke, Begutachtung von -

157 Far ben, Wirkung der - 273 Farbensinn 255ff. -- Untersuchung des - 176 Fleckfieber, Veränderungen der

Chorioidea bei -- 30 Follikelbildende Erreger 61 Foramen hyaloideae poste-

rius, symmetrisches - 1 Formwahrnehmungen 274

Gefäßhaut, Verfettung der - 61 Gefäßstreifen in der Netzhaut

und Ostitis fibrosa 63 Gehirnsklerose, tuberose 53, 158

- $\quad$ Fundusveränderungen bei tube-röser - 15

Gehirntumoren, Diagnose der -60

- $\quad$ Operationsanzeige bei -146 Germanin, Behandlung einer Co-

actatio conjunctivae mit --174 Geschichte der Augenheilkunde

128 ff. Gesichtsfeld 261 Gestaltswahrnehmungen 274 Glaskörper, Anomalien des -141 a

Beobachtungen an der vorde-

ren Grenzhaut des --- bei Cho-

rioideaabhebung 168 Glaskörperabhebung, hintere

ringförmige -224 
- $\quad$ Sichtbarmachung des Ringrisseseiner - mit freiem Auge 341

Glaukom 140, $180(\mathrm{Bb}$.

Vererbung des -325

juveniles - und Naevus flam-meus 171

- - und beiderseitiger Naevus flam-

meus 80 Gliome 52 Größenwahrnehmungen 274

Haradasche Krankheit 31 Hemeralopie 42 Heterochromie 118 Hochdruck, Retinopathie bei 39 Hornhaut s. Cornea. Hydrophthalmus congenitus

114 Hypertelorismus 123

Hypophyse, Tumoren der H.-

Gegend 60 Hypotonie, Erzeugung des Bildes

der akuten -344

Intravasaler Druck in der Retina 38 Iris, Entwicklung der - 113

- $\quad$ spontane Zysten der Hinter-fläche der - 93

Iritis, embryonale 124

- $\quad$ metastatische bei Spondyl-arthritis ankylopoetica 347

Katarakt, angeborene 119

- $\quad$ im Kindesalter und Poikilo-derma atrophicans 63

Kataraktoperation, Modifika-tion der extrakapsulären - 175

- $\quad$ Verhalten der vorderen Glas-körpermembran nach intrakap-sulärer - 343

Kolobome 116

-- Vererbung der $\cdot-321$

- $\quad$ ÄtiologiederMakula-K. 297, 323Kontaktgläser 167Kreuzung der Nervenbahnen und

die bilaterale Symmetrie 7

Lastexaugenbinde 29 Laurence-Biedlsches Syn-

drom 44 Lenticonus posterior, Vererbung des - 323 Leukämie, Veränderungen der

Netzhaut bei - 42 Lichtsinn 248ff. Lider, Anomalien der -- 121 —-Geschwülste der 132, 159,

166 Linse, Anomalien der 121, 140

- Bedeutung des Vitamin C. beiden biologischen Oxydationender $\quad$--172

-- Cholesterinkristalle in der - 281 Lochbildung, degenerative - der

Macula 44 Lupus erythematodes corneae

292 Lymphogranulomatosis in-

guinalis, Netzhautverände-

rungen bei - $-\mathbf{3} 36$ Macula lutea, Degeneration der

$-44.45$

- $\quad$ Ätiologie der Kolobome der -297, 323

Malaria, Veränderungen der Retina bei - 35

Membrana papillaris, persistie-rende 113, 119, 321

Meningenzephalitis haemor-rhagica, Erblindung bei - 35

Mikrometrie des Fundus 329

Mikrophthalmus 116, 323

- $\quad$ Pigmentzyste in der Pupille beiangeborenem a- 341

Sachregister

zu Band 88.

351 
Mißbildungen des Auges ti3ff.

Musculus orbicularis, Entfer-nung des Tarsalteiles des - zur Bekämpfung des Entropiums und der Trichiasis 100

Myopie, Vererbung der -326

- niyop. Aderhautdegeneration 32

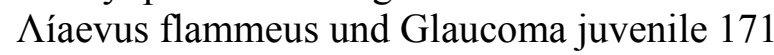

- beiderseitiger - und Glaukom 80

Naevus nonpigmentosus con-junctivae 173

Neophangläser 335

Nervenbahnen, Kreuzung der - und die bilaterale Symmetrie 7

Nervus opticus, Anomalien des

143

- $\quad$ Kompression des - durch dieCarotis interna 147

--· Endotheliom der Scheiden des

- $\quad 178$ Netzhaut s. RetinaNeuritis optica, Differentialdia-

gnose zwischen Pseudoneuritis-

Stauungspapille und - 303 Neuritis retrobulbaris nach

Fokalinfektion 165 Neurofibromatose der Ader-

haut 32 Nierenleiden, Retinopathie bei

- $\quad 39$ Nystagmus, familiärer 124

Oguchische Krankheit 42 Ophthalmia sympathica 139

- $\quad$ exogene und endogene Infektionbei --175

Ophthalmometer 339 Ophthalmoplegia interna 165 Optik, physiologische -- 248 (Ber.) physikalische -328 (Bericht)

- $\quad$ psychologische - 272 (Bericht)Orbita, Anomalien der - 144Ostitis fibrosa und

Gefäßstrei-

fen in der Netzhaut 63

Pachymeningitis haemorrha-gica interna mit Stauungs-papille 60

Papilla nervi optici, Tumorder

- $\quad 217$

Papilloma papillae lacrimalis

170 Paratrachom 164 Parinaudsche Krankheit 177 Pathologische Anatomie 132

(Bericht) Periphlebitis retinae 36

- neurologische Symptome bei -62, 164

Physikalisch-diätetische Be-handlung bei Augenkranken 348

Pigmentdegeneration der Netzhaut 43

Pigmentierung, Vererbung von Anomalien der - 324

Pigmentstreifen, angioide-des Fundus 45, 63

Pigmentzyste in der Pupille bei Mikrophthalmus congenitus 341

Poikiloderma atrophicans bei Katarakt im Kindesalter 63

Pseudoneuritis - Stauungspa-pille, Differentialdiagnose zwischen Neuritis optica und - $\mathbf{\square} 303$

Pseudotumor der Lider 64

Psoriasis 346

Ptosis, angeborene 123

Pupille, Verlagerungen und Schlitzform der $\mathbf{\square - \mathbf { m }} 118$

Pupillotonie u. Adiesche Krankheit 20 
Purkinjesches Phänomen 258

Radiumbestrahlung 151 Raumsinn 264 Raumwahrnehmungen 274

- $\quad$ zerebral bedingte Stoning der

- $\quad 163$

Raynaudsche Krankheit, Netz-hautveränderungen bei - 37

Reflexstreifen auf den Netzhaut-gefäßen 294

Refraktion, Bestimmung der -32811 .

Vererbung bei -325

Brechungsveränderungen bei diabetischen Veränderungen der

- $\quad$ 174Refraktionsbewegung des Auges,

angeborene - 123 Retina, Gefäßsystem der - 38

Anomalien der - 121, 141

Pathologie der -33 if.

Gefäßstreifen in der - 45, 63

Zirkulationsstörungen in der $\mathbf{m}$

173

- $\quad$ Reflexstreifen auf den Gefäßender - -294

- Beziehungen des intrakraniellen Druckes zu der Zirkulation der

- $\quad$ 287Retinitis centralis 38Retinitis circinata 45Retinitis exsudativa (Coats)

37 Retinitis punctata albescens

44 Röntgenbestrahlung 151 Röntgendiagnose bei unklaren

Sehnervenleiden 148 Röntgenstrahlen, Bedeutung

der - bei der Entstehung von

Mißbildungen 114 Rothmundsche Krankheit 63

352

Namenregister zu Band

Sarkom der Aderhaut 33

- der Aderhaut und die histo-

logischen Grundlagen des Au-

genspiegelbefundes 185 - metastatisches - der Uvea 86 Schielen, familiäres 124 Schüller-

Christiansche

Krankheit, Netzhautverände-

rungen bei der - 44 Schwangerschaftsniere, Re-

tinopathie bei - -40 Sehbahn, Anomalien der - 143 Sehnerv s. Nervus opticus Sehschärfe 264

Sehschwachenunterricht 283 Sehvermögen, zerebral bedingte

Störung des - 163 Sepsis, Verhalten der Retina bei

$-\quad 35$

Siderosis, Degeneration der Netz-

haut bei $\cdot-35$ Sinusitis als Ursache einer Ader-

hautdegeneration 30 Skiaskopie 339 Sklera, Anomalien der - 136 - - blaue 117

Spondylarthritis ankylopoe-

tica, metastatische Iritis bei -

347 Stauungspapille bei Pachyme-

ningitis haemorrhagicainterna 60 -Differentialdiagnose zwischen

Pseudoneuritis-St. und Neuritis 
optica 303 Strahlenschädigung der Retina

34 Symmetrie, bilaterale - - und

Kreuzung der Nervenbahnen 7 Synästhesie 272 Syphilis, Veränderungen der Cho-

rioidea bei - 30 Syringomyelie, Veränderungen

der Netzhaut bei -42

Tabak-Alkohol-Amblyopie, die Zerebrospinalflüssigkeit bei

- $\quad$ 293Teleangiektasia meningealis

179

Thrombangiitis obliterans, Netzhautveränderungen bei -36

Tiefensehen 264

Tortuositas vasorum 52

Trachom 13311.

- Entfernung des Tarsalteiles des Orbikularis zur Verhinderung des Rückfalles bei - durch Be-

kämpfung des Entropium und der Trichiasis 100

Tränendrüsenfistel, angeborene 123

Tränenröhrchen, außerordent-lich lange $\mathbf{~ - ~} 171$

Trichiasis, Entfernung des Tarsalteiles des Orbikularis zur Be-kämpfung der ·- 100

Tuberkulose, Veränderungen der Chorioidea bei - 31

Tuberkulosis uveo-parotidea 292

Tumoren der Aderhaut 32, 33

-- der Lider 132, 159, 166

Ulcus corneae, Ätiologie und

Therapie des eitrigen $-\cdot 346$ Ulcus serpens 162 Uvea, Anomalien der $\mathbf{-}-138$

Velonoskiaskopie 230, 338 Vena centralis retinae, Ver-

schluß der - 41, 160 Vererbung und Auge 3i9Íf. Vitamin C, Bedeutung des - bei

den biologischen Oxydationen

der Linse 172

Xanthomatosen 44

Zähne, Neuritis retrobulbaris nach

Fokalinfektion von den - 165 Zeitauffassung 279 Zerebrospinalflüssigkeit bei

Tabak-Alkohol-Amblyopie 293 Ziliargefäße, Zerreißung der hin-

teren - 166 Zyklektomie $75 \mathrm{Z}$ y s t e n , spontane - der Irishinter-

fläche 93

Namenregister zu Band 88.

Abe 31 Abel 274 Abraham 266 Accardi 41 Adler 265 Aglialoro 250 Agnello 123 Akiya 44

Aliquô-Mazzei 124 Allers 269 Allmaras 325 Amemiya 44 Amman 292 Amsler 34 Anargyros 93 Ancona 119

A. Apelmans 122 Archangelskij 42 Arkin 117, 319 Armstrong 112 Arruga33,47,48,50,5i Ask 33 Aubaret 20, 327 Averbach 48 